\title{
Decoupled bifunctional air electrodes for high-performance hybrid lithium-air batteries
}

\author{
Longjun Li and Arumugam Manthiram* \\ Materials Science and Engineering Program \& Texas Materials Institute \\ The University of Texas at Austin \\ Austin, TX 78712, USA
}

\begin{abstract}
Lithium-air batteries have become appealing in recent years, but one of the major challenges is the large overpotential associated with the oxygen reduction reaction (ORR) and oxygen evolution reaction (OER). Tremendous efforts have been made on developing highly active and durable catalysts to lower the overpotentials for ORR and OER. In addition to the intrinsic activity and stability of the catalysts, construction of the air electrodes plays an important role in the overall performance of the air electrodes. Accordingly, three kinds of electrode configurations are herein compared: single, combined, and decoupled bifunctional air electrodes. The decoupled design of the air electrodes shows the best performance in terms of both the discharge and charge performance compared to the other configurations.
\end{abstract}

Keywords: Lithium-air batteries; hybrid cells; bifunctional catalysts; electrode configurations; decoupled design

*Corresponding author. Tel: +1-512-471-1791; fax: +1-512-471-7681.

E-mail address: manth@austin.utexas.edu (A. Manthiram) 


\section{Introduction}

Hybrid Li-air batteries, in which the lithium-metal anode in a nonaqueous electrolyte is separated from the air cathode in an aqueous catholyte by a solid electrolyte membrane, show attractive properties for applications like electrical vehicles or grid-energy storage [1-4]. They offer several advantages such as high cell voltage, high energy density, stability in ambient air, and reversibility in aqueous catholytes. However, the development of hybrid Li-air batteries is still at its infant stage. Many efforts have been made on extending the cycle life of rechargeable hybrid Li-air batteries. The research directions include the development of highly active and stable bifunctional catalysts [5-8], highly porous cathodes for better air diffusion [9, 10], suitable aqueous catholytes to maintain the stability of the solid electrolyte [11-13], and highly conductive solid electrolytes that are stable with lithium-metal anode and aqueous catholytes $[14,15]$. Noble-metal catalysts were first applied in hybrid Li-air batteries as a proof of concept $[9,11,16]$. Thin carbon nanotube (CNTs) buckypaper cathodes, which intertwined in a horizontal direction and formed large open channels for air diffusion, could reduce the Pt loading to as low as 5 wt. \% [9]. However, the noble-metal catalysts are scarce and expensive, limiting the practical applications.

Carbon-based catalysts are good candidates for the oxygen reduction reaction (ORR) during the discharge process in alkaline solutions, considering their low cost and high activity $[17,18]$. There are two examples of using pure carbon materials as 
air cathodes in alkaline catholyte based hybrid Li-air batteries. Li et al. [10] recently reported that less populated, vertically aligned nitrogen-doped carbon nanotube arrays (CNTAs) with dislocated graphene stacking can be grown directly onto carbon fiber papers and investigated them as air cathodes for hybrid Li-air batteries with alkaline electrolytes. They found that these CNTAs exhibit high electrocatalytic activity, which is comparable to that of $20 \mathrm{wt} . \% \mathrm{Pt} / \mathrm{C}$ during battery test [10]. Zhou et al. [7] utilized an all graphene-based catalyst in hybrid Li-air batteries. They found that a high-temperature heat-treatment increases the stability of graphene at high voltage by decreasing the oxygen functional groups and planar defects in graphene. However, the loss of oxygen functional groups and planar defects also decrease the ORR catalytic activity of carbon materials [19]. As already proved, carbon has very low oxidation potential, which makes it vulnerable during the high-voltage charge process. One promising way to suppress the oxidation of carbon at high voltage is to couple it with metal oxides [20], which are more active and stable to the oxygen evolution reaction (OER). The most attractive catalysts with this approach are the cobalt oxides (e.g., $\mathrm{CoO}$ or $\mathrm{Co}_{3} \mathrm{O}_{4}$ ) coupled with graphene or carbon nanotubes [21-23]. However, the connection between the oxides and carbon support is subject to loss under the high charge potential, leading to degradation of the catalysts [24]. Therefore, the rechargeable Li-air batteries require alternative strategies to realize acceptable cycle life.

To avoid the involvement of the ORR catalyst layer in the oxidizing charge process, the concept of decoupling the ORR and OER electrodes was first explored in 
metal hydride-air batteries in 1995 and demonstrated in hybrid Li-air batteries recently $[1,25,26]$. However, a systematic study of how the air electrode configurations affect the activity and stability of cell performance with the same catalysts is still lacking. We present here such an investigation by directly growing spinel $\mathrm{NiCo}_{2} \mathrm{O}_{4}$ nanoflakes onto nickel foam as a decoupled OER catalyst and a comparison of the performance with that of conventional $\mathrm{NiCo}_{2} \mathrm{O}_{4}$ nanoflakes powder. Three kinds of air-electrode configurations are compared here, each containing the $\mathrm{Pt} / \mathrm{C}$ as the ORR catalyst and $\mathrm{NiCo}_{2} \mathrm{O}_{4}$ nanoflakes (NCONF) as the OER catalyst. The conventional configuration (single) contains both Pt/C and NCONF in one single catalyst layer. Both catalysts are loaded onto a hydrophobic carbon-fiber paper to undergo the reducing discharge and oxidizing charge processes. The second configuration (decoupled) is to separate the ORR and OER functions into two different electrodes. While the $\mathrm{Pt} / \mathrm{C}$ is loaded onto a hydrophobic carbon-fiber paper to play the role of $\mathrm{ORR}, \mathrm{NiCo}_{2} \mathrm{O}_{4}$ nanoflakes are directly grown onto a hydrophilic nickel foam (NCONF@Ni) to play the role of OER. The cathode is switched between the ORR and OER electrodes during discharge and charge. The third configuration (combined) is to combine the current collectors of the decoupled ORR and OER electrodes during cell operation. In this way, the cathode need not be switched between the two electrodes, leading to a convenient cell handling.

We find that the decoupled configuration displays the best overall ORR and OER performances. This is because the ORR and OER require quite different electrochemical environments, which are fulfilled by separating these two functions 
into two independent electrodes with different properties. The $\mathrm{Pt} / \mathrm{C}$ catalyst was loaded onto a hydrophobic carbon paper to maximize the three-phase boundary for ORR. The decoupled design also avoids the involvement of $\mathrm{Pt} / \mathrm{C}$ in the oxidizing OER process. The $\mathrm{NiCo}_{2} \mathrm{O}_{4}$ nanoflakes were grown onto a three-dimensional (3-D) porous nickel foam and totally immersed in the electrolyte to ensure full contact of the OER catalyst with the electrolyte. Each nanoflake was directly connected to the conductive nickel foam substrate to achieve high catalytic efficiency.

\section{Experimental}

\section{Synthesis}

To synthesize the $\mathrm{NiCo}_{2} \mathrm{O}_{4}$ nanoflakes powder, $0.5 \mathrm{mmol}$ of $\mathrm{Ni}\left(\mathrm{NO}_{3}\right)_{2} \bullet 6 \mathrm{H}_{2} 0, \quad 1$ mmol of $\mathrm{Co}\left(\mathrm{NO}_{3}\right)_{2} \cdot 6 \mathrm{H}_{2} \mathrm{O}$, and $3 \mathrm{mmol}$ hexamethylene-tetramine were added into a mixture of deionized water and ethanol $(30 \mathrm{~mL}, 2: 1 \mathrm{v} / \mathrm{v})$, resulting in a mole ratio of $1: 2: 6$. A Teflon-lined stainless-steel autoclave was then used to conduct a hydrothermal treatment with the above solution at $90{ }^{\circ} \mathrm{C}$ for $10 \mathrm{~h}$. After the autoclave cooled down, the greenish $\mathrm{Ni}-\mathrm{Co}$ precursor precipitated in the solution was collected by centrifugation, washed with deionized water and ethanol for several times, and dried at $80{ }^{\circ} \mathrm{C}$ overnight. $\mathrm{NiCo}_{2} \mathrm{O}_{4}$ nanoflakes powder was finally obtained by firing the obtained dry powder at $320^{\circ} \mathrm{C}$ for $2 \mathrm{~h}$ in air.

To synthesize the $\mathrm{NiCo}_{2} \mathrm{O}_{4}$ nanoflakes on nickel foam [27], a pre-cleaned nickel foam $(2 \mathrm{~cm} \times 4 \mathrm{~cm})$ was immersed in the above solution when conducting the hydrothermal treatment. After the solution was cooled down, the nickel foam was covered with a layer of greenish $\mathrm{Ni}-\mathrm{Co}$ precursor, preferably on the side facing down. 
After cleaning, the nickel foam was subjected to the same hydrothermal treatment second time with the other side facing down. The nickel foam deposited with the Ni-Co precursor was finally washed with copious amount of water and ethanol, dried at $80{ }^{\circ} \mathrm{C}$ overnight, and annealed at $320^{\circ} \mathrm{C}$ for $2 \mathrm{~h}$ in air.

\section{Characterization}

X-ray diffraction (XRD) was carried out with a Philips X-ray diffractometer equipped with $\mathrm{CuK} \alpha$ radiation from 10 to $70^{\circ}$ at a scan rate of $0.02^{\circ} \mathrm{s}^{-1}$. The morphology and microstructural characterizations were carried out with a FEI Quanta 650 SEM and JEOL 2010F transmission electron microscope (TEM). X-ray photoelectron spectroscopy (XPS) analysis was conducted with a Kratos Analytical spectrometer. The deconvolution of the XPS spectrum was performed using CasaXPS software with Gaussian-Lorentzian functions and a Shirley background.

\section{Half cell test}

The cycling performance of the air electrodes was tested in a home-made three-electrode half cell, in which $0.5 \mathrm{M} \mathrm{LiOH}+1 \mathrm{M} \mathrm{LiNO}_{3}, \mathrm{a} \mathrm{Hg} / \mathrm{HgO}$ electrode, and a platinum flag were used, respectively, as the electrolyte, reference electrode, and counter electrode. The method to prepare the air electrode has been reported before elsewhere [11].

\section{Full cell test}

A home-made PTFE layered battery mould was used to carry out the full cell test. The anode side was assembled in an argon-filled glove box and then combined 
with the cathode side in air. The anode side consisted of a nickel foam current collector, a lithium metal foil, and the organic carbonate electrolyte $\left(1 \mathrm{M} \mathrm{LiPF}_{6}\right.$ in ethylene carbonate (EC) / diethylcarbonate (DEC) $(1: 1 \mathrm{v} / \mathrm{v}))$, which has been commonly used as an anode electrolyte in other hybrid Li-air batteries $[6,9,11]$. The cathode side consisted of $2 \mathrm{~mL}$ of $0.5 \mathrm{M} \mathrm{LiOH}+1 \mathrm{M} \mathrm{LiNO}_{3}$ solution as the catholyte, a hydrophobic carbon fiber paper $(0.76 \times 0.76 \mathrm{~cm})$ containing $1.0 \mathrm{mg} \mathrm{cm}{ }^{-2} \mathrm{Pt} / \mathrm{C}(60$ wt. \%) + $1.0 \mathrm{mg} \mathrm{cm}^{-2} \mathrm{NiCo}_{2} \mathrm{O}_{4}$ nanoflakes powder, and a platinum mesh current collector. The catholyte composition of $\mathrm{LiOH}+\mathrm{LiNO}_{3}$ was proved effective during charge and discharge in alkaline electrolyte Li-air batteries by Li et al. [10]. For the combined and decoupled configurations, a hydrophobic carbon-fiber paper $(0.76 \times$ $0.76 \mathrm{~cm}$ ) containing $1.0 \mathrm{mg} \mathrm{cm}^{-2} \mathrm{Pt} / \mathrm{C}$ acted as the ORR electrode, while $1.0 \mathrm{mg} \mathrm{cm}^{-2}$ $\mathrm{NiCo}_{2} \mathrm{O}_{4}$ nanoflakes directly grown onto the nickel foam acted as the OER electrode. A solid electrolyte $\left(\mathrm{Li}_{1+x+y} \mathrm{Ti}_{2-\mathrm{x}} \mathrm{Al}_{\mathrm{x}} \mathrm{P}_{3-\mathrm{y}} \mathrm{Si}_{\mathrm{y}} \mathrm{O}_{12}\right.$ or LTAP, $0.15 \mathrm{~mm}$ thick, $\sigma=1 \times 10^{-4} \mathrm{~S}$ $\mathrm{cm}^{-1}, 0.76 \times 0.76 \mathrm{~cm}$, OHARA Inc., Japan) was used as the separator. The cathode side of the hybrid Li-air cell was purged with water-saturated air during operation to suppress the evaporation of water from the catholyte [28].

Discharge-charge experiments were conducted on an Arbin BT 2000 battery cycler. Two independent Arbin channels were used to collect the discharge and charge data alternatively with a 5-minute rest time between each discharge and charge period. To avoid switching the cable in practical applications, a diode can be connected with the ORR electrode to block the charge current without influencing the discharge current. Polarization curves were recorded on a VoltaLab PGZ 402 
potentiostat by sweeping the potential at $10 \mathrm{mV} \mathrm{s}^{-1}$.

\section{Results and discussion}

\section{Characterizations of different OER electrodes}

As we can see from the SEM images in Fig. 1a and b, the synthesized $\mathrm{NiCo}_{2} \mathrm{O}_{4}$ was composed of spherical particles of micron size with a flower-like texture. As we zoom in, we find that these spherical particles are actually made of interconnected nanoflakes. These nanoflakes were analyzed by TEM as shown in Fig. 1c. They are very thin as they appear in the low-magnification TEM image and contain large amounts of mesopores all over the surface. The polycrystalline nature of these nanoflakes was characterized by the selective area electron diffraction (SAED) pattern shown in Fig. 1c. The X-ray diffraction (XRD) pattern is shown in Fig. 1d. The broad peaks indicate small crystallite size, which is in agreement with the TEM observation. The detected peaks match well with those of spinel $\mathrm{NiCo}_{2} \mathrm{O}_{4}$.

The detailed surface composition and oxidation state of the nanoflakes were analyzed by XPS as shown in Fig. 2. The full survey indicates the presence of Ni 2p, Co 2p, O 1s, and C 1s in Fig. 2a [29-31]. With a Gaussian fitting method, the high-resolution Ni 2p spectrum was best fitted with two spin-orbital doublets, corresponding to $\mathrm{Ni}^{2+}$ and $\mathrm{Ni}^{3+}$, and two shakeup satellites (Fig. 2b) [31]. Similarly, the spectrum of Co $2 \mathrm{p}$ was best fitted with two spin-orbital doublets of $\mathrm{Co}^{2+}$ and $\mathrm{Co}^{3+}$, and two shakeup satellites (Fig. 2c) [30]. The high-resolution O 1s spectrum contains four oxygen contributions denoted as O1, O2, O3, and O4 in Fig. 2d [29]. Component $\mathrm{O} 1$ represents metal-oxygen bonds. The component $\mathrm{O} 2$ is typical of oxygen in 
hydroxyl groups, which may be due to the oxygen atoms of surface hydroxyl groups. The resolved $\mathrm{O} 3$ component is associated with oxygen in low coordination at the surface, which is common for nanoparticles. The $\mathrm{O} 4$ contribution corresponds to multiplicity of physisorbed- and chemisorbed water on or near the surface.

The detailed characterizations of $\mathrm{NiCo}_{2} \mathrm{O}_{4}$ nanoflakes grown onto nickel foam are shown in Fig. 3. As we can see from the low- and high-magnification SEM images in Fig. 3a and b, a dense layer of $\mathrm{NiCo}_{2} \mathrm{O}_{4}$ nanoflakes has been grown on the nickel foam, which can greatly increase the contact surface area between the OER electrode and the catholyte. These nanoflakes are of micron size in diameter and very thin as they appear under TEM shown in Fig. 3c. They possess the same polycrystalline and mesoporous feature as the powder material shown in Fig. 1. From the XPS analysis shown in Fig. $d$ to f, we can see the surface composition and oxidation state of NCONF@Ni are the same as those of NCONF powder material. Thus, any differences in catalytic activity and stability should be contributed by the different configuration of the air electrodes.

The activity of NCONF@Ni was tested in a three-electrode half-cell with a $\mathrm{Hg} / \mathrm{HgO}$ reference electrode, a platinum flag counter electrode, and an electrolyte made of $0.5 \mathrm{M} \mathrm{LiOH}+1 \mathrm{M} \mathrm{LiNO}_{3}$. The performance is shown in Fig. 4. As a comparison, the OER activities of a blank nickel foam and NCONF powder were also measured with the same half-cell configuration. For the NCONF powder, it was sonicated in an ethanol solution containing LITHion binder (10 wt. \%, Ion Power, USA) and air sprayed onto a hydrophobic carbon paper. The loading of all the 
catalysts was controlled to be $1.0 \mathrm{mg} \mathrm{cm}^{-2}$. The LITHion content in the air electrodes was 20 wt. \%. As we can see from Fig. 4, the nickel foam has the lowest current density with an onset potential at $0.75 \mathrm{~V}$ vs. $\mathrm{Hg} / \mathrm{HgO}$. The $\mathrm{NiCo}_{2} \mathrm{O}_{4}$ nanoflakes powder has much better OER catalytic activity than nickel foam with an onset potential at around $0.7 \mathrm{~V}$ vs. $\mathrm{Hg} / \mathrm{HgO}$ due to the high intrinsic OER activity of spinel $\mathrm{NiCo}_{2} \mathrm{O}_{4}$ and the large surface area contributed by the mesopores. A small bump can be seen at around $0.6 \mathrm{~V}$ vs. $\mathrm{Hg} / \mathrm{HgO}$ corresponding to the $\mathrm{M}-\mathrm{O} / \mathrm{M}-\mathrm{O}-\mathrm{OH}$ redox couple ( $\mathrm{M}$ represents $\mathrm{Ni}$ and $\mathrm{Co}$ ) [32]. After growing the $\mathrm{NiCo}_{2} \mathrm{O}_{4}$ nanoflakes onto the nickel foam, we can see that the catalytic activity is further improved. The use of NCONF@Ni has the following advantages: first, each $\mathrm{NiCo}_{2} \mathrm{O}_{4}$ nanoflake is electrically connected to the current collector, maximizing the utilization of $\mathrm{NiCo}_{2} \mathrm{O}_{4}$ nanoflakes in the OER process; second, like the pure carbon 3-D structured cathode recently reported by Li et al. [10], the 3-D porous structure of $\mathrm{Ni}$ foam increases the active sites for OER, enhances the mass transfer of reactants or products, and keeps smooth electron pathways for the rapid electrochemical reactions; third, this direct "grown on" design enables a strong connection between the catalyst and nickel foam matrix to endure any detaching force coming from the rising oxygen bubbles during the OER process. In addition, as each $\mathrm{NiCo}_{2} \mathrm{O}_{4}$ nanoflake is connected to the nickel foam current collector, binders and conductive additives are eliminated. 


\section{Electrochemical performance of the three configurations of bifunctional air}

\section{electrodes}

The configuration of the conventional single bifunctional air electrode and corresponding cycling voltage profiles in the half cell are shown in Fig. 5a. The loading of the ORR catalyst Pt/C (60 wt. \%) is $1.0 \mathrm{mg} \mathrm{cm}^{-2}$ and that of the OER catalyst $\left(\mathrm{NiCo}_{2} \mathrm{O}_{4}\right.$ nanoflakes powder $)$ is $1.0 \mathrm{mg} \mathrm{cm}^{-2}$. The advantage of this configuration is the simplicity for manufacturing and installation in the batteries. However, the electrode needs to be engineered to reach an intermediate state of being hydrophobic for the ORR and hydrophilic for the OER. However, some ORR catalyst particles are inevitably flooded to lose the ORR catalytic activity and some OER catalyst particles are inevitably blocked from the electrolyte to lose the OER catalytic activity. More importantly, the ORR catalyst needs to undergo the highly oxidizing OER conditions during the high-voltage charge process. Unfortunately, most ORR catalyst and carbon support are vulnerable to high-voltage, which seriously degrades the air electrode upon cycling $[33,34]$. Thus, we can observe the fast degradation of the ORR performance of the air electrode upon cycling in Fig. 5a.

One way to avoid the highly corrosive OER conditions on the ORR catalyst and carbon support is to separate these two functions into two independent electrodes as shown in Fig. 5b. The NCONF@Ni works as the OER electrode and the loadings of both catalysts remain $1 \mathrm{mg} \mathrm{cm}^{-2}$. The $\mathrm{Pt} / \mathrm{C}$ catalyst was loaded onto a hydrophobic carbon paper to maximize the three-phase boundary for ORR. The $\mathrm{NiCo}_{2} \mathrm{O}_{4}$ nanoflakes were grown onto a 3-D porous nickel foam and totally immersed in the 
electrolyte to ensure the full contact of the OER catalyst and electrolyte. The cathode needs to be switched between the ORR and OER electrodes during discharge and charge. We can observe much improved cycling performance compared to the conventional air electrode.

As a comparison, the combined design is shown in Fig. 5c. The current collectors of the two electrodes are connected to ensure the convenient handling during cell operation. However, the ORR electrode still participates in the high-voltage charge process, which increases the Pt degradation and carbon oxidation [35]:

$$
\mathrm{C}+2 \mathrm{H}_{2} \mathrm{O}=\mathrm{CO}_{2}+4 \mathrm{H}^{+}+4 \mathrm{e}^{-} \mathrm{E}_{298 \mathrm{k}}=0.207 \mathrm{~V} \text { vs. RHE }
$$

Thus, we can still observe fast degradation of the ORR performance upon cycling, as seen in Fig. 5c. However, the OER performance is very stable due to the binder- and carbon-free NCONF@Ni contributing to the charge process. Overall, a decoupled ORR and OER electrode design is beneficial for both the discharge and charge of the air electrode.

\section{Electrochemical performance of the hybrid Li-air batteries with different configurations of bifunctional air electrodes}

In order to show the advantages of the decoupled design of the bifunctional air electrodes in hybrid Li-air batteries over the conventional electrode, the cycling performances of the two cells with $1.0 \mathrm{mg} \mathrm{cm}^{-2} \mathrm{Pt} / \mathrm{C}(60 \mathrm{wt} . \% \mathrm{Pt} / \mathrm{C})+1 \mathrm{mg} \mathrm{cm}^{-2}$ NCONF or $1.0 \mathrm{mg} \mathrm{cm}^{-2} \mathrm{Pt} / \mathrm{C}$ (60 wt. \% Pt/C) + $1 \mathrm{mg} \mathrm{cm}^{-2}$ NCONF@Ni bifunctional air electrodes were tested. The discharge and charge voltage profiles of the two cells 
at a current density of $0.5 \mathrm{~mA} \mathrm{~cm}^{-2}$ at room temperature are compared in Fig. 6. Pt/C is used as the ORR catalyst because it is a stable ORR catalyst so that the observed difference in battery efficiency and cyclability can be attributed to the OER catalyst. As we can observe in Fig. 6, the initial round-trip overpotential based on the end voltage is $0.75 \mathrm{~V}$ for $\mathrm{Pt} / \mathrm{C}+\mathrm{NCONF}$ and $0.81 \mathrm{~V}$ for Pt/C + NCONF@Ni, attributing to a voltaic efficiency of, respectively, $80.4 \%$ and $79.0 \%$. The observed slightly smaller round-trip overpotential of $\mathrm{Pt} / \mathrm{C}+\mathrm{NCONF}$ compared to that of $\mathrm{Pt} / \mathrm{C}+$ NCONF@Ni in the first cycle is because both Pt/C and NCONF in the conventional single air electrode can participate in the discharge and charge processes, leading to a lower effective current density per catalyst weight. As the cycle number increases, the gap between the discharge and charge voltage plateaus increases due to the slow degradation of both the ORR and OER catalysts during the cycling process [6]. However, we can observe that the degradation process of the novel decoupled cell configuration is much slower than that of the conventional configuration. After 50 cycles, the round-trip overpotential of the hybrid $\mathrm{Li}$-air batteries with $\mathrm{Pt} / \mathrm{C}+\mathrm{IrO}_{2}$ increased to $1.24 \mathrm{~V}$ compared to $0.93 \mathrm{~V}$ with Pt/C + NCONF@ Ni, corresponding to a voltaic efficiency of, respectively, $70.4 \%$ and $76.4 \%$. The cycling performance shown above proves that the decoupled bifunctional air electrodes can achieve similar activity but much better stability than the conventional single bifunctional air electrodes with the same catalysts utilized [6]. The high stability of the decoupled air electrodes is due to (i) the avoidance of the high-voltage charge process for the ORR air electrode and (ii) the high electrochemical and mechanical stability of the $\mathrm{NiCo}_{2} \mathrm{O}_{4}$ 
nanoflakes on the nickel foam during the OER process.

\section{Conclusions}

In summary, three configurations of bifunctional air electrodes have been compared in terms of their activity and durability. The decoupled ORR and OER air electrode configuration achieved activity similar to but stability much better than the conventional electrode. Mesoporous $\mathrm{NiCo}_{2} \mathrm{O}_{4}$ nanoflakes were directly grown onto the nickel foam to form a 3-D binder- and carbon-free OER air electrode, offering high OER activity and good electrochemical stability during the OER process. The additional OER electrode also avoids the involvement of ORR catalyst layer in the high-voltage OER process, eliminating the degradation of ORR catalyst layer by the carbon support degradation and catalyst detachment, and thereby leading to superior cycle performance of the hybrid Li-air batteries.

\section{Acknowledgements}

This work was supported by the U.S. Department of Energy, Office of Basic Energy Sciences, Division of Materials Sciences and Engineering under award number DE-SC0005397. 


\section{References}

[1] H. Zhou, Y. Wang, H. Li, P. He, ChemSusChem 3 (2010) 1009-1019.

[2] T. Zhang, N. Imanishi, Y. Takeda, O. Yamamoto, Chemistry Letters 40 (2011) 668-673.

[3] R. Black, B. Adams, L.F. Nazar, Advanced Energy Materials 2 (2012) 801-815.

[4] Y. Wang, H. Zhou, Journal of Power Sources 195 (2010) 358-361.

[5] L. Li, A. Manthiram, Journal of Materials Chemistry A 1 (2013) 5121-5127.

[6] K. Huang, Y. Li, Y. Xing, Electrochimica Acta 103 (2013) 44-49.

[7] E. Yoo, H. Zhou, ACS Nano 5 (2011) 3020-3026.

[8] W. Yang, J. Salim, S. Li, C. Sun, L. Chen, J.B. Goodenough, Y. Kim, Journal of Materials Chemistry 22 (2012) 18902-18907.

[9] Y. Li, K. Huang, Y. Xing, Electrochimica Acta 81 (2012) 20-24.

[10] Y. Li, Z. Huang, K. Huang, D. Carnahan, Y. Xing, Energy \& Environmental Science 6 (2013) 3339-3345.

[11] L. Li, X. Zhao, A. Manthiram, Electrochemistry Communications 14 (2012) 78-81.

[12] T. Zhang, N. Imanishi, Y. Shimonishi, A. Hirano, Y. Takeda, O. Yamamoto, N. Sammes, Chemical Communications 46 (2010) 1661-1663.

[13] Y. Shimonishi, T. Zhang, N. Imanishi, D. Im, D.J. Lee, A. Hirano, Y. Takeda, O. Yamamoto, N. Sammes, Journal of Power Sources 196 (2011) 5128-5132.

[14] L. Puech, C. Cantau, P. Vinatier, G. Toussaint, P. Stevens, Journal of Power Sources 214 (2012) 330-336.

[15] Y. Shimonishi, A. Toda, T. Zhang, A. Hirano, N. Imanishi, O. Yamamoto, Y. Takeda, Solid State Ionics 183 (2011) 48-53.

[16] T. Zhang, N. Imanishi, S. Hasegawa, A. Hirano, J. Xie, Y. Takeda, O. Yamamoto, N. Sammes, Journal of the Electrochemical Society 155 (2008) A965-A969.

[17] L. Li, A. Manthiram, Advanced Energy Materials (2014), doi: 10.1002/aenm.201301795.

[18] L. Li, S.-H. Chai, S. Dai, A. Manthiram, Energy \& Environmental Science (2014), doi: 10.1039/C4EE00814F.

[19] S. Wang, S. Dong, J. Wang, L. Zhang, P. Han, C. Zhang, X. Wang, K. Zhang, Z. Lan, G. Cui, Journal of Materials Chemistry 22 (2012) 21051-21056.

[20] K. Huang, Y. Li, L. Yan, Y. Xing, RSC Advances 4 (2014) 9701-9708.

[21] Y. Liang, Y. Li, H. Wang, J. Zhou, J. Wang, T. Regier, H. Dai, Nature Materials 10 (2011) 780-786.

[22] Y. Liang, H. Wang, P. Diao, W. Chang, G. Hong, Y. Li, M. Gong, L. Xie, J. Zhou, J. Wang, T.Z. Regier, F. Wei, H. Dai, Journal of the American Chemical Society 134 (2012) 15849-15857.

[23] S. Guo, S. Zhang, L. Wu, S. Sun, Angewandte Chemie International Edition 51 (2012) 11770-11773. 
[24] Y. Li, M. Gong, Y. Liang, J. Feng, J.-E. Kim, H. Wang, G. Hong, B. Zhang, H. Dai, Nature Communications 4 (2013) 1805.

[25] T. Sakai, T. Iwaki, Z. Ye, D. Noreus, Journal of the Electrochemical Society 142 (1995) 4040-4045.

[26] P. Stevens, G. Toussaint, G. Caillon, P. Viaud, P. Vinatier, C. Cantau, O.

Fichet, C. Sarrazin, M. Mallouki, ECS Transactions 28 (2010) 1-12.

[27] G. Zhang, X.W. Lou, Advanced Materials 25 (2013) 976-979.

[28] L. Li, X. Zhao, Y. Fu, A. Manthiram, Physical Chemistry Chemical Physics 14 (2012) 12737-12740.

[29] J.F. Marco, J.R. Gancedo, M. Gracia, J.L. Gautier, E. Ríos, F.J. Berry, Journal of Solid State Chemistry 153 (2000) 74-81.

[30] J.G. Kim, D.L. Pugmire, D. Battaglia, M.A. Langell, Applied Surface Science 165 (2000) 70-84.

[31] J. Li, S. Xiong, Y. Liu, Z. Ju, Y. Qian, ACS Applied Materials \& Interfaces 5 (2013) 981-988.

[32] H. Wang, Q. Gao, L. Jiang, Small 7 (2011) 2454-2459.

[33] S.-E. Jang, H. Kim, Journal of the American Chemical Society 132 (2010) 14700-14701.

[34] S. Zhang, X.-Z. Yuan, J.N.C. Hin, H. Wang, K.A. Friedrich, M. Schulze, Journal of Power Sources 194 (2009) 588-600.

[35] E. Guilminot, A. Corcella, F. Charlot, F. Maillard, M. Chatenet, Journal of the Electrochemical Society 154 (2007) B96-B105. 


\section{Figure Captions}

Figure 1. (a) and (b) SEM image, (c) TEM image, and (d) XRD pattern of the $\mathrm{NiCo}_{2} \mathrm{O}_{4}$ nanoflakes obtained by annealing the precipitated $\mathrm{Ni}-\mathrm{Co}$ precursor in solution.

Figure 2. XPS analysis of the $\mathrm{NiCo}_{2} \mathrm{O}_{4}$ nanoflakes: (a) survey, (b) $\mathrm{Ni} 2 \mathrm{p}$, (c) Co $2 \mathrm{p}$, and (d) O 1s spectra.

Figure 3. Characterizations of the $\mathrm{NiCo}_{2} \mathrm{O}_{4}$ nanoflakes on nickel foam: (a) and (b)

SEM images, (c) TEM image and SAED pattern, (d) XPS survey spectrum, (e) XPS Ni 2p peak, and (f) XPS Co 2p peak.

Figure 4. Polarization curves of the different OER electrodes.

Figure 5. Cycling performances of the various configurations of bifunctional air electrodes at $2.0 \mathrm{~mA} \mathrm{~cm}^{-2}$ : (a) single, (b) decoupled, and (c) combined bifunctional air electrodes.

Figure 6. Cycling performance of the hybrid Li-air batteries with (a) the single Pt/C + NCONF air electrode or (b) decoupled Pt/C + NCONF@ Ni at 0.5 mA $\mathrm{cm}^{-2}$. 

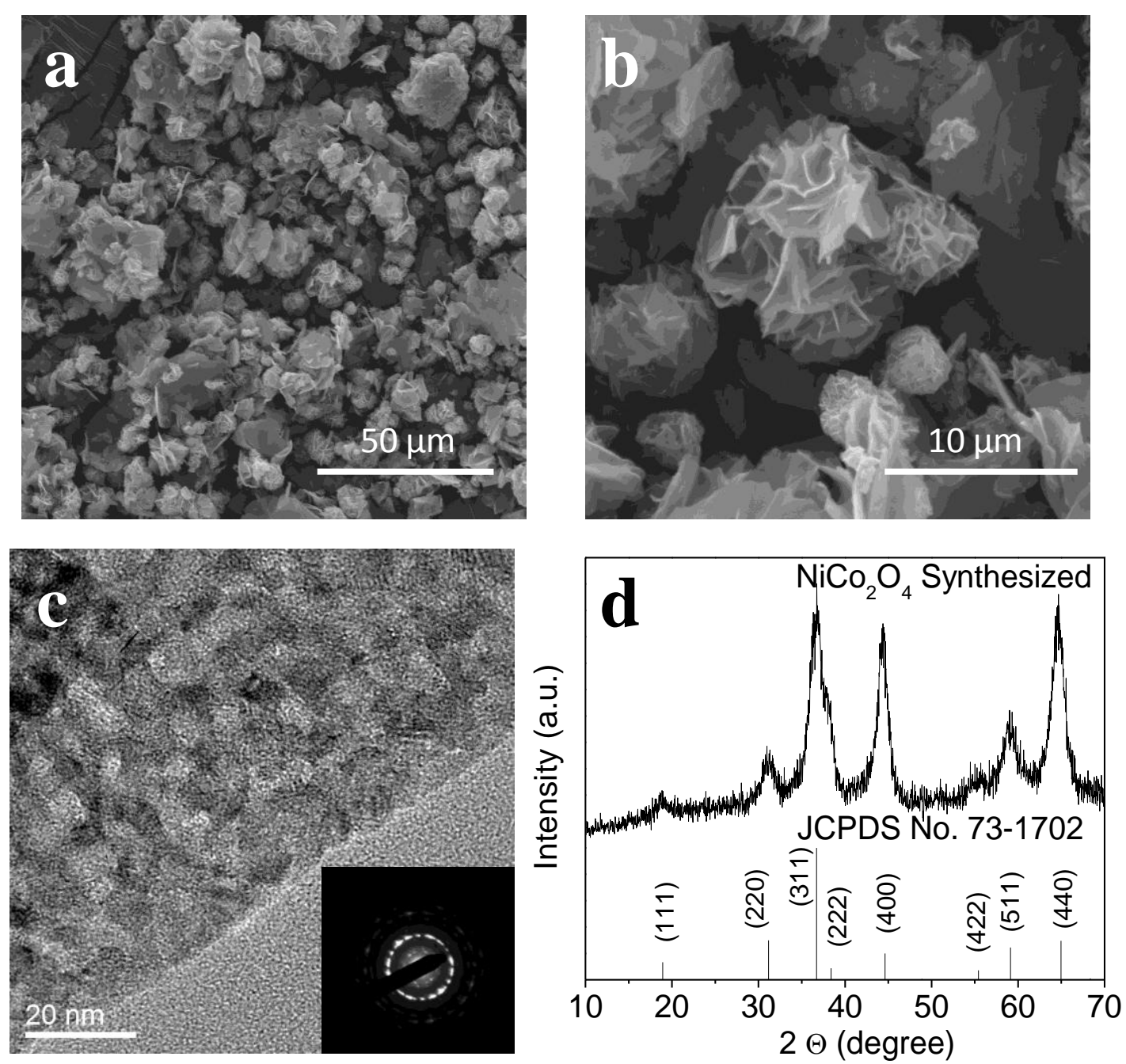

Figure 1 

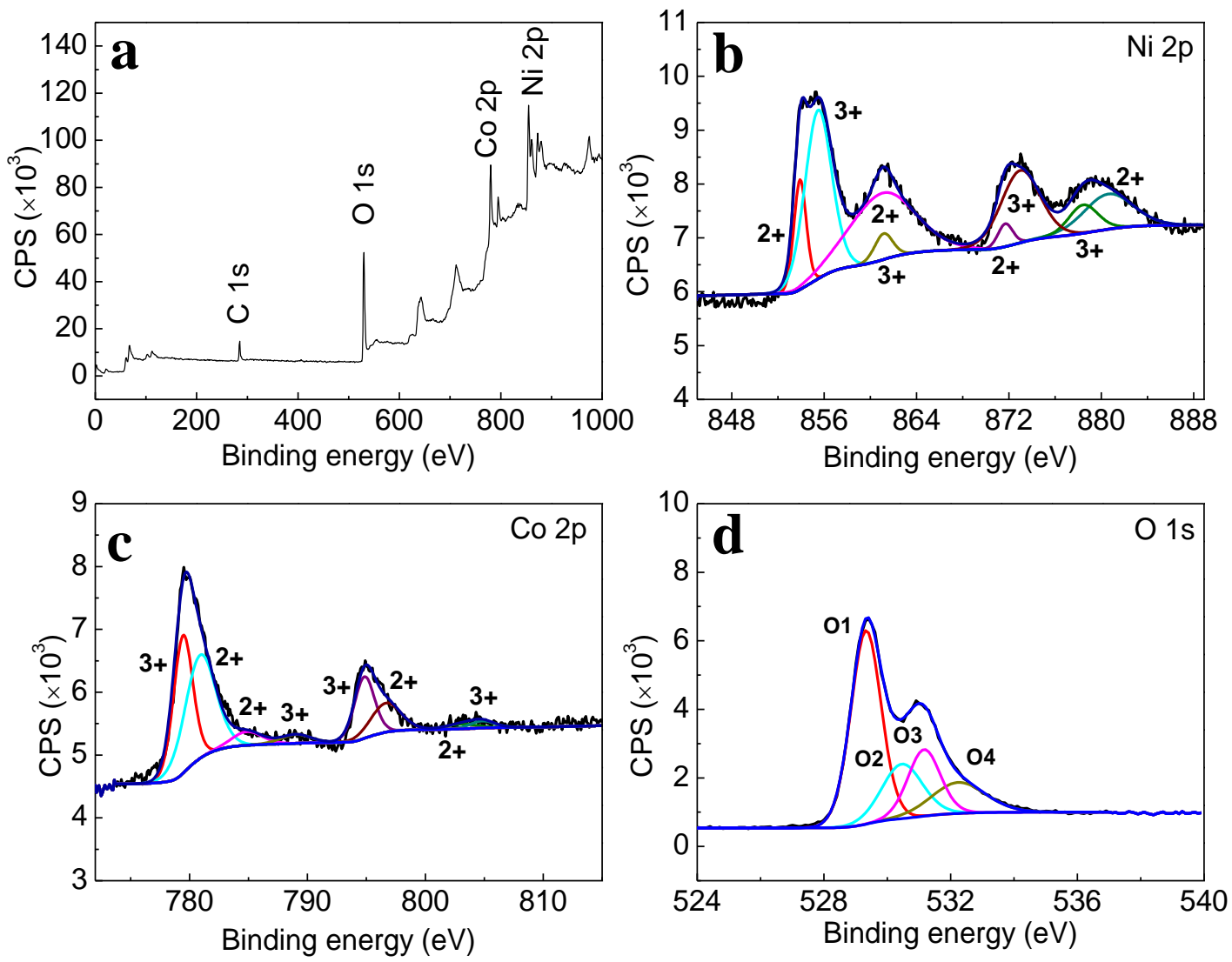

Figure 2 

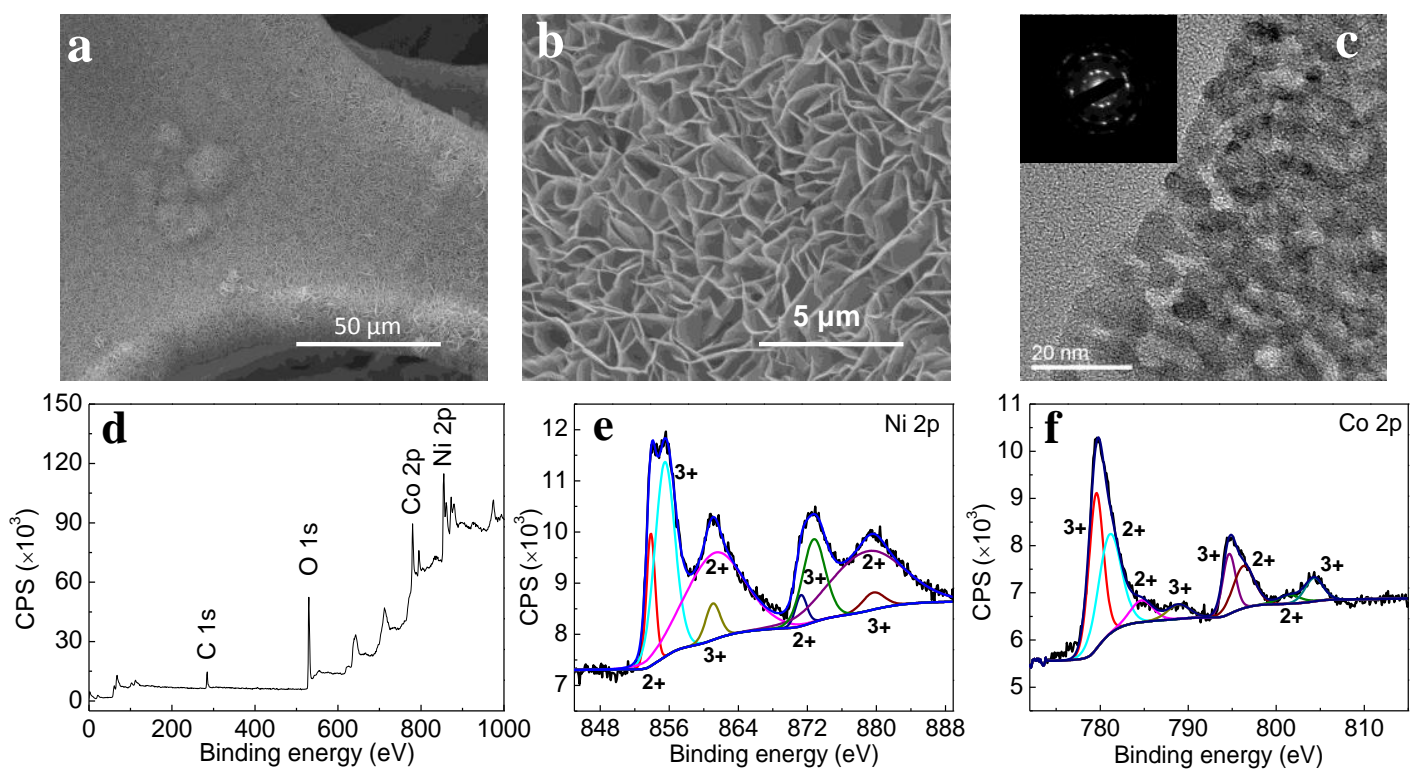

Figure 3 


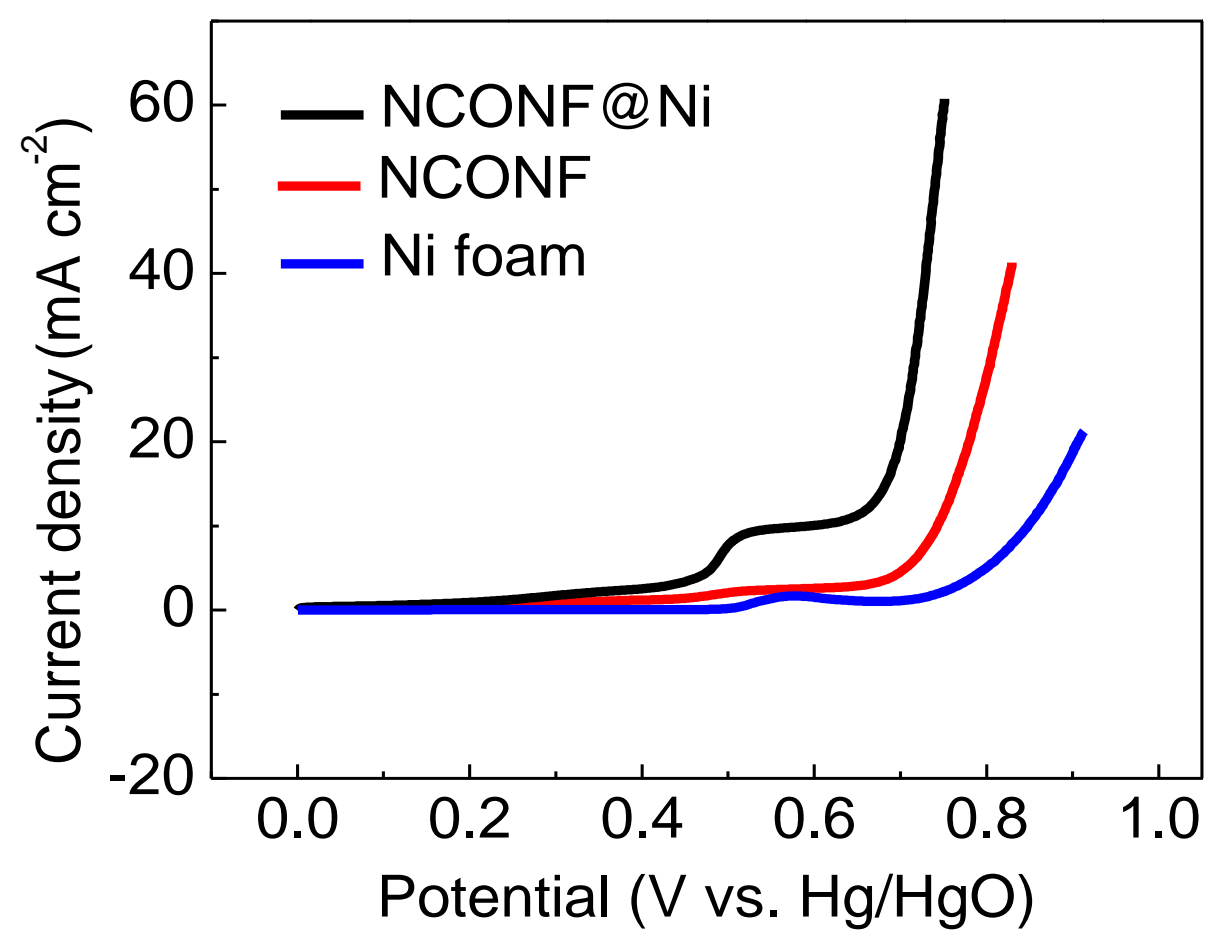

Figure 4 

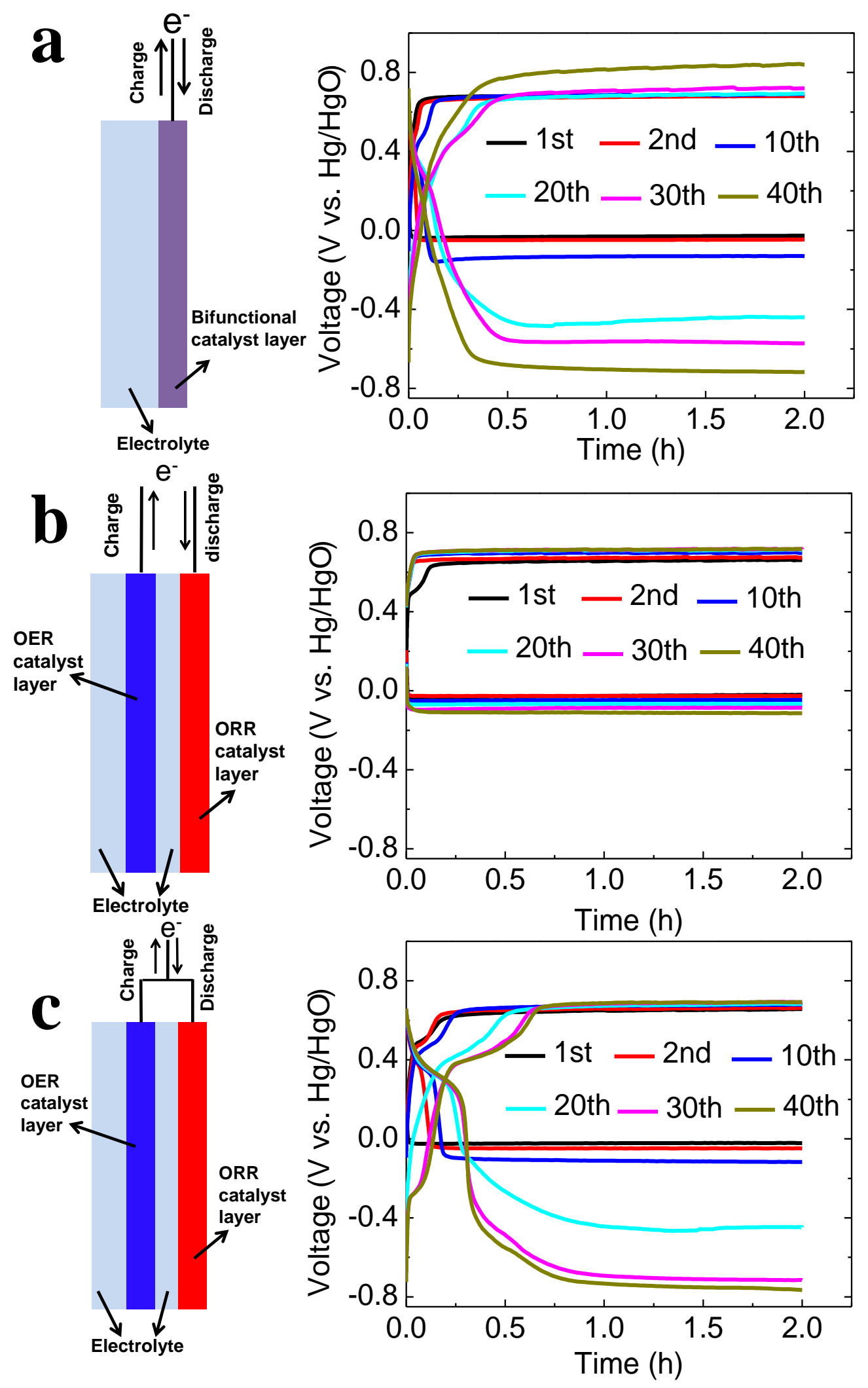

Figure 5 

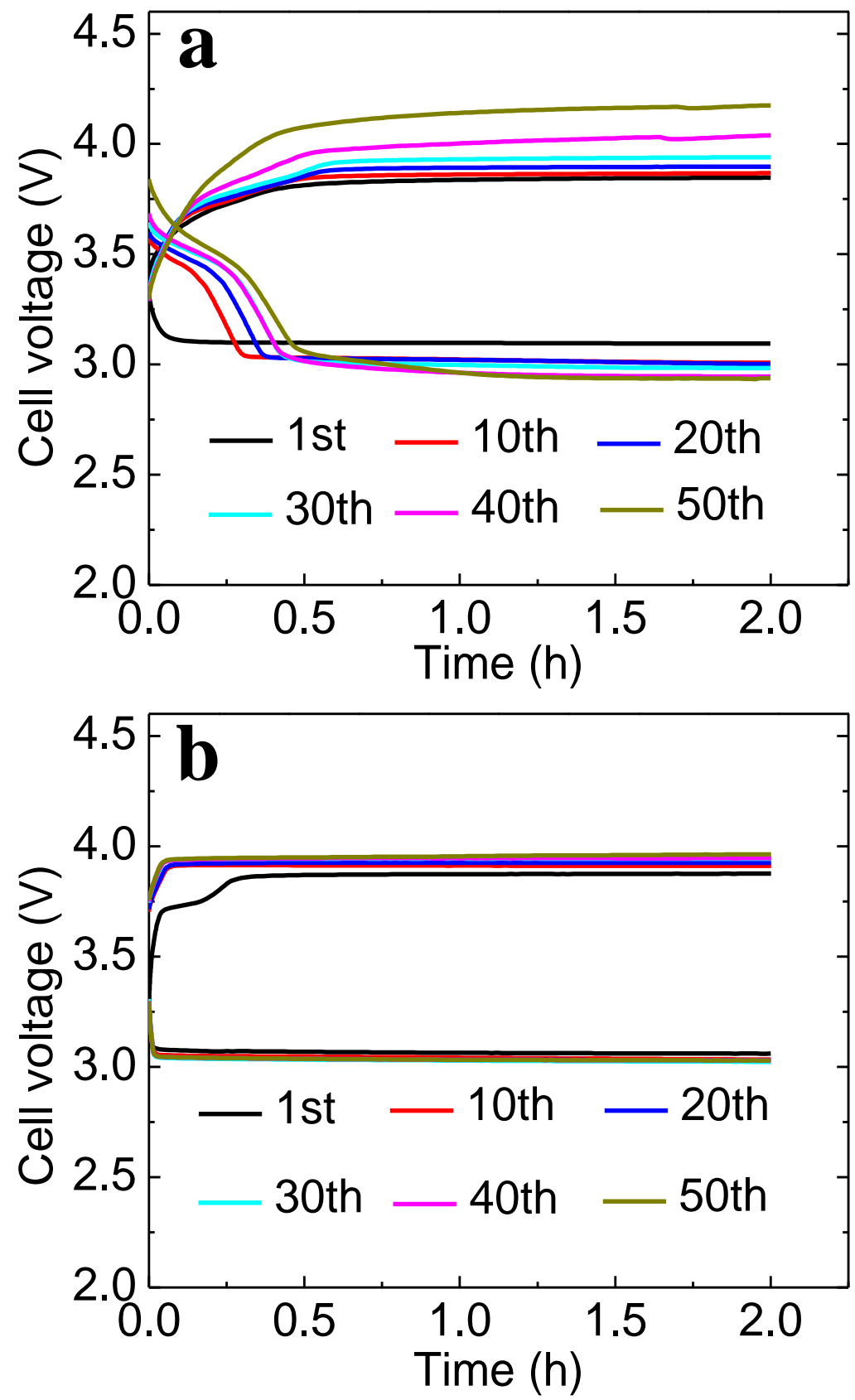

Figure 6 


\section{Vitae}

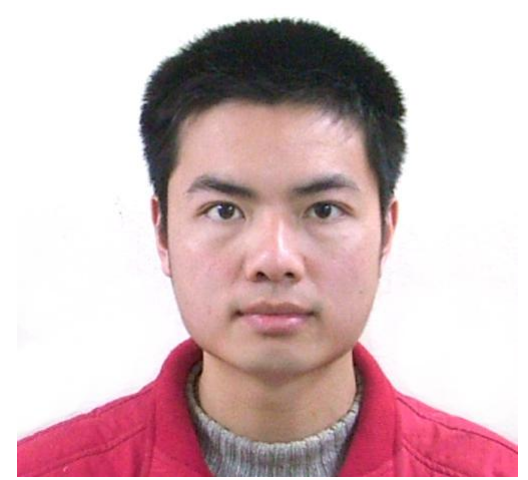

Longjun Li received his B. Eng. degree from

Department of Materials Science and Engineering at Huazhong University of Science and Technology in 2010. He is now pursuing his Ph.D. studies under the supervision of Prof. Arumugam Manthiram at the University of Texas at Austin (UT Austin). His research interest focuses on hybrid $\mathrm{Li}$-air batteries and related materials.

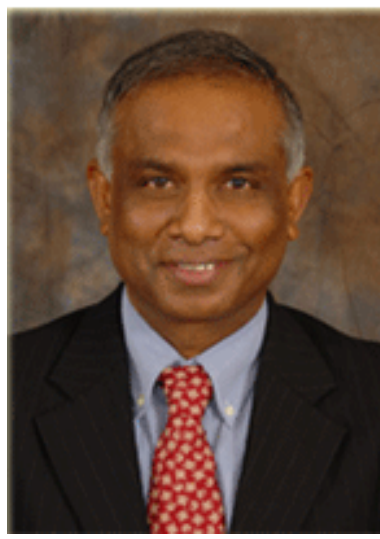

Arumugam Manthiram is a Professor and holder of the Joe C. Walter Chair in Engineering in the Materials Science and Engineering Graduate Program and Department of Mechanical Engineering at The University of Texas at Austin. He is also the Director of the Texas Materials Institute and the Materials Science and Engineering Program. His research interests are in the area of materials for rechargeable batteries, fuel cells, and solar cells, including novel synthesis approaches for nanomaterials and nanocomposites. He has authored more than 550 publications including more than 470 journal articles. See www.me.utexas.edu/ manthiram for further details. 


\section{Graphical Abstract}

A decoupled design of oxygen reduction reaction and oxygen evolution reaction electrodes in hybrid Li-air batteries is found to show activity similar to but stability much better than the conventional single electrodes.
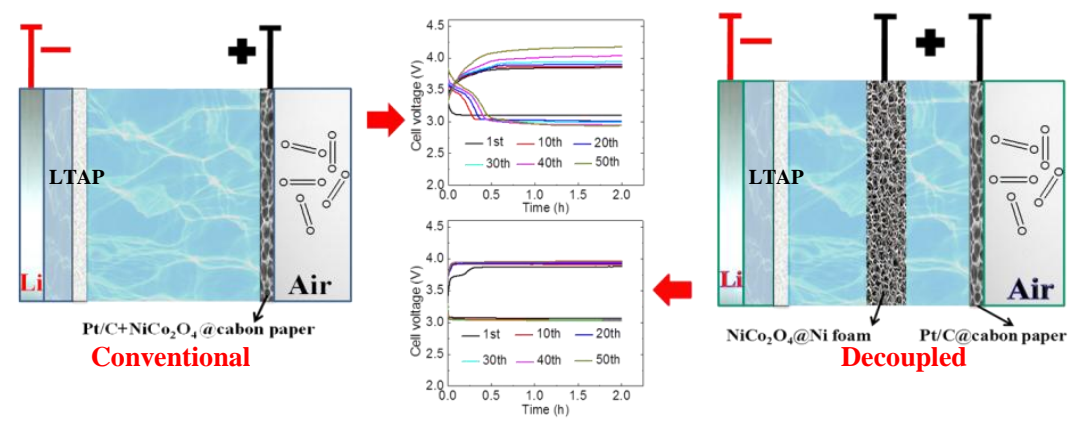